\title{
Dynamic Characteristics of Hanging Truss Having Shape Memory Alloy Wires
}

\author{
Xuan Zhang ${ }^{1}$, Kazuyuki Hanahara ${ }^{2} \&$ Yukio Tada $^{1}$
}

${ }^{1}$ Graduate School of System Informatics, Kobe University, Kobe, Japan
${ }^{2}$ Faculty of Science and Engineering, Iwate University, Iwate, Japan
Correspondence: Xuan Zhang, Graduate School of System Informatics, Kobe University, Kobe, Japan. E-mail: cs22-
tyou@ @stu.kobe-u.ac.jp

Received: August 23, 2017

Accepted: September 25, 2017

Online Published: November 1, 2017

doi:10.5539/mer.v7n2p6

URL: https://doi.org/10.5539/mer.v7n2p6

\begin{abstract}
In this study, we discuss the dynamics of a type of hanging truss structural system consisting of rigid and wire members, part of which are SMA (shape memory alloy) wires. This kind of truss structure has the capability of vibration isolation and absorption. Characteristics of zero compressive stiffness of wire members, SMA wire members and hanging configuration of the structure itself contribute to the effect of vibration isolation. The hysteretic loop of SMA wires plays a significant role in vibration attenuation. Mathematical models for this kind of dynamic problem are developed. Calculation process is introduced to take into account the mechanical characteristics of SMA and wire members. Dynamic characteristics are discussed; simultaneously, the effects of vibration isolation and attenuation have been confirmed. On the basis of the numerical calculations, advantages of combinations of various types of wire members, including the truss units having no bracing wires have been demonstrated.
\end{abstract}

Keywords: dynamic behavior, hanging truss, natural frequency, shape memory alloy wire

\section{Introduction}

There are a large number of studies on reducing the influence of environmental vibration. For example, in order to isolate the substructure beam from vibration, variable stiffness damped absorbers are used (Patil \& Awasare, 2016). A damper with porous anisotropic outer ring and the compressed oily layer is put forward for the purpose of reducing the vibration impact (Akhverdiev \& Mukutadze, 2016).

There are two ways for reducing the influence of environmental vibration. One is the vibration isolation that aims to cut off the transmission of vibration motion to the target object in consideration. The other is the vibration suppression that aims to reduce or attenuate the vibration itself. Several studies on vibration isolation taking advantage of the behavior of the pendulum have been conducted in recent years. Seismic isolation systems using translational pendulum are free from influence of the weight of the structure they bear and functionally stable (Tatemichi et al., 2004). The results obtained by Sanap et al. (2014) show that the friction pendulum system works effectively in limiting the building responses during excitation due to earthquakes (Sanap et al., 2014). The multi-suspended pendulum isolation system developed by Narita et al. (2000) works well concerning vibration isolation (Narita et al., 2000).

Vibration attenuation is expected to be achieved by various types of vibration energy absorbers. Shape memory alloy material is a kind of smart material that has been studied for its conspicuous characteristics of shape memory effect in relatively low temperature condition and pseudo-elasticity in relatively high temperature condition. There are many researches concerning passive vibration control using SMA material. For instance, in the case of dealing with environmental vibration attenuation by means of a structural system, the effect is not remarkably significant based only on the hysteretic characteristic of the SMA material. Combinations of the SMA material and viscoelastic material demonstrate noticeable result of vibration attenuation (De Lima et al., 2014). Utilization of the damping capacity of martensite phase of SMA as well as the re-centering capability of austenite phase of SMA was studied for the purpose of reaching the best feasible performance by arranging different states of the SMA materials as the bracing elements of the structures (Mortazavi et al., 2013). For the purpose of obtaining the maximum energy dissipation capability, the optimal configurations of the combination of SMA wires and energy-absorbing struts of the structures are attained (Yang et al., 2010). Besides, there are many studies on vibration attenuation by SMA material that works as bracing elements (Tamai \& Kitagawa, 2002; McCormick et al., 2010; Song et al., 2006; Omar, 2014). 
A hanging truss having SMA wires is expected to demonstrate the effects of vibration isolation by the characteristic of pendulum and vibration attenuation by the nonlinear hysteretic loop of SMA material. The dynamic behavior of this kind of structure is dealt with as a nonlinear dynamic problem. A number of researches on natural frequency problems for nonlinear dynamics have been reported. Dynamic behavior and vibration control of a tensegrity structure have been modeled and discussed in (Ali \& Smith, 2010), where the modal analysis of that structure is conducted as well by means of numerical simulation and experiment. In their analysis, the free vibration result reveals the existence of a beat phenomenon in tensegrity time history response. For the purpose of reducing the vibration while the variable geometry truss moves a target payload from one point to another as a space manipulator, an optimization of motion plan has been studied taking consideration of the natural frequencies (Senba et al., 2009). An advantage of a tensegrity-inspired tower with regard to frequency tuning by shape morphing using shape memory alloy actuators has been explored (dos Santos et al., 2015). The frequency tuning reduces the accelerations throughout the structure up to about 80 percent.

In the current study, we deal with a truss structural system in hanging type that consists of rigid and wire members; part of the wire members are of SMA. Dynamics calculation of truss structural system having various types of truss members (Hanahara et al., 2016) are adopted. We discuss about the dynamic characteristics of the hanging truss structures having SMA wires and ordinary wires with the time history of natural frequency. This type of truss structure becomes a mechanism in the case of less stiff members required to maintain the statical determinacy, due to the compressive force on wires and/or due to the removal of wires. However, owing to the influence of the gravitational force, stability of this mechanism can be guaranteed in many situations. We develop a mathematical model of the dynamic problem in consideration. For the purpose of dealing with the nonlinearities of this dynamic problem, a time integration method coupled with nonlinear iterative method is introduced. The effect of hanging configuration of the truss, as well as the effects of mechanical properties of SMA and ordinary wires are demonstrated respectively. We confirm the following characteristic benefits in dynamic behavior of the hanging truss: the vibration isolation capability taking advantage of the mechanism condition and the vibration attenuation ability due to hysteretic loop of the SMA wires. It should be noted in addition that the ordinary wires have constant tensile stiffness while the stiffness of SMA wires changes in accordance with their stress and histories of the routes of the corresponding constitutive models. Adequate combinations of these factors are expected to exhibit excellent vibration isolation and attenuation effects. Variation of natural frequency of the truss structure is discussed in connection with the effect of vibration isolation taking advantage of the variable stiffness of truss structure due to the variable stiffness of SMA wires.

\section{Dynamic Model of Truss Structural System}

A brief introduction is made on the pseudo-elastic constitutive equation of SMA material in one dimension. The dynamic equation of the truss structural system having SMA wires is formulated; its time history of the natural frequency is also taken into account.

\subsection{Constitutive Equation of SMA}

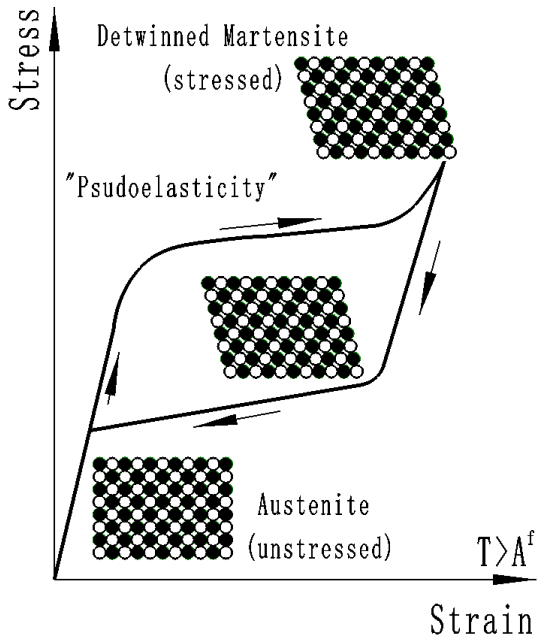

(a) Conceptual illustration

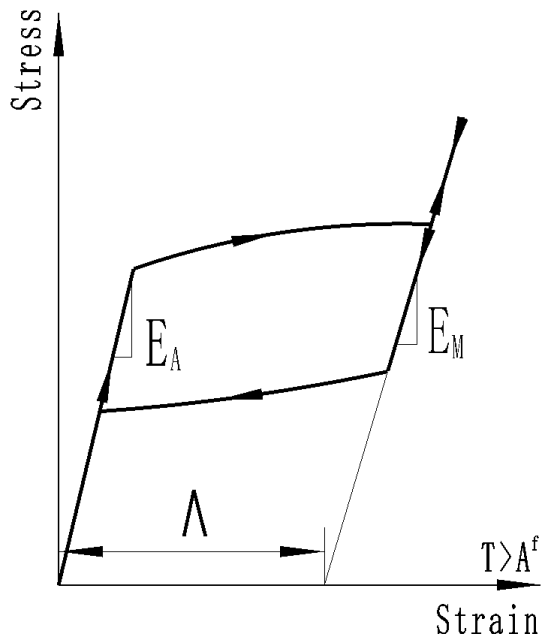

(b) Introduced model

Figure 1. Pseudo-elasticity of SMA

Figure 1(a) shows the stress-strain relationship of the pseudo-elastic constitutive model of SMA material considering the 
crystalline structures of austenite phase and martensite phase. It is impractical to adopt the mathematical model in such a manner; thus, a pseudo-elastic piecewise mathematic model as shown in Figure 1(b) is introduced.

In Figure 1(b), parameters $E_{A}$ and $E_{M}$ are the Young's moduli of the SMA material in austenite and martensite phases respectively, without phase transformation; parameter $\Lambda$ is the maximum phase transformation strain. The total strain of the SMA material can be decomposed as follows:

$$
\epsilon=\epsilon^{e}+\epsilon^{t}=\epsilon^{e}+\Lambda \lambda
$$

in this equation, parameter $\epsilon^{e}$ is the elastic strain, parameter $\epsilon^{t}$ is the phase transformation strain and parameter $\lambda$ is the martensite phase volume fraction. Constitutive relation of SMA is written as:

$$
\sigma=E \epsilon^{e}=E[\epsilon-\Lambda \lambda]
$$

where $\sigma$ is the stress of the SMA material, $E$ is the current Young's modulus that can be determined by the current martensite phase volume fraction, and the Young's moduli in the martensite and austenite phases (Auricchio \& Sacco, 1997):

$$
E=\frac{E_{A} E_{M}}{E_{M}+\left(E_{A}-E_{M}\right) \lambda}
$$

Incremental equation is derived from this equation as follows:

$$
\Delta \sigma=E[\Delta \epsilon-\Lambda \Delta \lambda]
$$

As the relation between the increments of the volume fraction of the martensite phase $\Delta \lambda$ and the total strain $\Delta \epsilon$ is assumed to be proportional, the following equation can be derived:

$$
\Delta \lambda=H \Delta \epsilon
$$

where the proportional coefficient $H$ is the tangent coefficient. Substituting this equation into Equation (4), the incremental form of the constitutive equation is obtained as follows:

$$
\Delta \sigma=E_{T} \Delta \epsilon, \quad E_{T}=E[1-H \Lambda]
$$

We can see that at the stages of phase transformation, the tangent elastic coefficient $E_{T}$ changes with the martensite phase volume fraction. The proportional coefficient $H$ can be calculated by Equation (4) and the linearization of the discrete evolution equation with the active phase transformation written in residual form (Hu, 2013).

\subsection{Equation of Motion}

The dynamics calculation is carried out on the basis of the formulations in (Hanahara et al., 2016). However, as the introduction of the constitutive relation of SMA wire, the nonlinear force of SMA wire member in that dynamic equation should be modified. On the basis of the result (Lagoudas et al., 2001), the stress of SMA wire member is calculated as:

$$
\sigma=E \epsilon^{e}=E\left(\epsilon-\epsilon^{t}\right)
$$

Substituting the SMA wire member stress into the dynamic equation, and taking the constant gravitational force $\boldsymbol{G}$ into consideration, the dynamic equation of motion is derived as:

$$
\boldsymbol{M}_{C} \ddot{\boldsymbol{D}}_{C}+\boldsymbol{K}_{C} \boldsymbol{D}_{C}=\boldsymbol{F}_{X C}+\boldsymbol{J}_{C}^{T} \boldsymbol{q}+\boldsymbol{G}_{C}
$$

where subscript ' $C^{\prime}$ indicates that these vectors and matrices contain the elements corresponding to the nodes fixed on the support ceiling. The matrices and vectors in Equation (8) are as follows: $\boldsymbol{M}_{C}$ is the nodal inertia matrix, $\boldsymbol{F}_{X C}$ is the external nodal force due to the environmental vibration of support ceiling, $\boldsymbol{q}$ is the axial member force due to the deformation of SMA wire members, taking account of the influence of phase transformation for the SMA wire members and $\boldsymbol{G}_{C}$ is the equivalent nodal force due to the gravitational acceleration acting on the structural mass. Total nodal displacement vector taking into account of the deformation is denoted as $\boldsymbol{D}_{C}$. Matrix $\boldsymbol{J}_{C}=\partial \boldsymbol{L} / \partial \boldsymbol{P}_{C}$ is the coordinate transformation matrix which transforms member values to the corresponding nodal values. Vector $\boldsymbol{L}$ is the current member length vector and vector $\boldsymbol{P}_{C}$ is the nodal positions vector taking into account of both of member deformation and support ceiling movement. Matrix $\boldsymbol{K}_{C}$ is the nodal stiffness matrix that can be expressed as:

$$
\boldsymbol{K}_{C}=\boldsymbol{J}_{C}^{T} \boldsymbol{K}_{r} \boldsymbol{J}_{C}
$$

where $\boldsymbol{K}_{r}=\operatorname{diag}\left[E_{1} A_{1} / l_{1}, \ldots, E_{N} A_{N} / l_{N}\right]$, and $A_{i}, l_{i}$ and $N$ are the cross sectional area, the natural length of the $i$ th member and the total number of the members, respectively. 


\subsection{Natural Frequency}

We obtain the sub-matrices $\boldsymbol{K}$ and $\boldsymbol{M}$ of stiffness and inertia matrices $\boldsymbol{K}_{C}$ and $\boldsymbol{M}_{C}$, by extracting the elements corresponding to the nodes that are not fixed on the support ceiling. On the basis of these matrices, modal analysis of the truss structural system is conducted by the following equation:

$$
\boldsymbol{K}-\omega_{k}^{2} \boldsymbol{M}=\mathbf{0}
$$

$\omega_{k}$ denotes the $k$ th angular natural frequency of the truss structural system; the corresponding natural frequency is obtained as $f_{k}=\omega_{k} / 2 \pi$. Owing to the material nonlinearity of the SMA wire members and the geometric nonlinearity of the truss structural system itself, the stiffness matrix $\boldsymbol{K}$ is time dependent and so do the natural frequencies. In this research, the first natural frequency $f_{1}$ at time $t$ is calculated and evaluated.

\section{Numerical Calculation}

In the same manner as the previous section, the dynamic equation of the truss structural system is rewritten by paying attention to the nodes that are not fixed on the support ceiling as:

$$
\boldsymbol{M} \ddot{\boldsymbol{D}}+\boldsymbol{K} \boldsymbol{D}=\boldsymbol{F}+\boldsymbol{J}^{T} \boldsymbol{q}
$$

In order to deal with the nonlinearity of this kind of dynamic problem, the nonlinear iterative method is introduced (Thai $\& \mathrm{Kim}, 2011)$. The fundamental equations for the numerical integration are derived in the following sections.

\subsection{Newmark $\beta$ Method Considering Nonlinear Iteration}

In the dynamic equation (11), as the coordinate transformation matrix $\boldsymbol{J}$ contains the geometric nonlinear characteristic and the member force vector $\boldsymbol{q}$ depends on the nodal displacement as well as the nonlinear characteristics of SMA and wire members, the transformed nodal force vector $\boldsymbol{J}^{T} \boldsymbol{q}$ has to be examined in accordance with the dynamics calculation. We introduce the following iterative approach to calculate the state at time $t+\Delta t$ based on the state at time $t$. The equation of motion (11) to be satisfied at $t+\Delta t$ is approximated by using the stiffness matrix $\boldsymbol{K}^{(t)}$ and force vector $\left(\boldsymbol{J}^{(t)}\right)^{T} \boldsymbol{q}^{(t)}$ at $t$ as:

$$
\boldsymbol{M} \ddot{\boldsymbol{D}}^{(t+\Delta t)}+\boldsymbol{K}^{(t)} \boldsymbol{D}^{(t+\Delta t)}=\boldsymbol{F}^{(t+\Delta t)}+\left(\boldsymbol{J}^{(t)}\right)^{T} \boldsymbol{q}^{(t)}
$$

On the basis of the Newmark $\beta$ method, the nodal displacement $\boldsymbol{D}^{(t+\Delta t)}$ and its velocity $\dot{\boldsymbol{D}}^{(t+\Delta t)}$ at $t+\Delta t$ are represented in terms of the acceleration $\ddot{\boldsymbol{D}}^{(t+\Delta t)}$. By substituting these representations into Equation (12) and solving the obtained linear equation, $\ddot{\boldsymbol{D}}^{(t+\Delta t)}$ is calculated. Once $\ddot{\boldsymbol{D}}^{(t+\Delta t)}$ is known, $\boldsymbol{D}^{(t+\Delta t)}$ and $\dot{\boldsymbol{D}}^{(t+\Delta t)}$ are calculated based on the representations. The matrix and vector at $t+\Delta t, \boldsymbol{K}^{(t+\Delta t)}$ and $\left(\boldsymbol{J}^{(t+\Delta t)}\right)^{T} \boldsymbol{q}^{(t+\Delta t)}$ are accordingly updated based on $\boldsymbol{D}^{(t+\Delta t)}$. Force error vector due to the approximation at this moment can be evaluated as:

$$
\boldsymbol{F}_{\text {err }}^{(t+\Delta t)}=\boldsymbol{F}^{(t+\Delta t)}+\left(\boldsymbol{J}^{(t+\Delta t)}\right)^{T} \boldsymbol{q}^{(t+\Delta t)}-\left(\boldsymbol{M} \ddot{\boldsymbol{D}}^{(t+\Delta t)}+\boldsymbol{K}^{(t+\Delta t)} \boldsymbol{D}^{(t+\Delta t)}\right)
$$

For the purpose of dealing with the nonlinearity of this kind of dynamic problem, it is necessary to update $\boldsymbol{D}^{(t+\Delta t)}, \boldsymbol{K}^{(t+\Delta t)}$ and $\left(\boldsymbol{J}^{(t+\Delta t)}\right)^{T} \boldsymbol{q}^{(t+\Delta t)}$ several times to suppress the force error vector by introducing the corresponding incremental vectors as follows:

$$
\boldsymbol{M}\left(\ddot{\boldsymbol{D}}^{(t+\Delta t)}+\mathrm{d} \ddot{\boldsymbol{D}}\right)+\boldsymbol{K}^{(t+\Delta t)}\left(\boldsymbol{D}^{(t+\Delta t)}+\mathrm{d} \boldsymbol{D}\right)=\boldsymbol{F}^{(t+\Delta t)}+\left(\boldsymbol{J}^{(t+\Delta t)}\right)^{T}\left(\boldsymbol{q}^{(t+\Delta t)}+\mathrm{d} \boldsymbol{q}\right)
$$

Re-arranging the above equation by taking Equation (13) into consideration, we obtain the following equation:

$$
\boldsymbol{M} \mathrm{d} \ddot{\boldsymbol{D}}+\boldsymbol{K}^{(t+\Delta t)} \mathrm{d} \boldsymbol{D}-\left(\boldsymbol{J}^{(t+\Delta t)}\right)^{T} \mathrm{~d} \boldsymbol{q}=\boldsymbol{F}_{e r r}^{(t+\Delta t)}
$$

To denote the update time as $\mu$, the fundamental equation of displacements of Newmark $\beta$ method at update times $\mu$ and $\mu-1$ can be written as follows respectively.

$$
\begin{aligned}
& \boldsymbol{D}^{(t+\Delta t, \mu)}=\boldsymbol{D}^{(t)}+\Delta t \dot{\boldsymbol{D}}^{(t)}+\Delta t^{2}\left[\left(\frac{1}{2}-\beta\right) \ddot{\boldsymbol{D}}^{(t)}+\beta \ddot{\boldsymbol{D}}^{(t+\Delta t, \mu)}\right] \\
& \boldsymbol{D}^{(t+\Delta t, \mu-1)}=\boldsymbol{D}^{(t)}+\Delta t \dot{\boldsymbol{D}}^{(t)}+\Delta t^{2}\left[\left(\frac{1}{2}-\beta\right) \ddot{\boldsymbol{D}}^{(t)}+\beta \ddot{\boldsymbol{D}}^{(t+\Delta t, \mu-1)}\right]
\end{aligned}
$$

According to the above two equations, the relationship between $\mathrm{d} \ddot{\boldsymbol{D}}$ and $\mathrm{d} \boldsymbol{D}$ can be derived as:

$$
\mathrm{d} \ddot{\boldsymbol{D}}=\frac{1}{\beta \Delta t^{2}} \mathrm{~d} \boldsymbol{D}
$$

Different from the original Newmark $\beta$ method, we choose the displacement as the primary variable. On the basis of Equation (17) and the linear relation (24) between the correction in member force $\mathrm{d} \boldsymbol{q}$ and the correction in nodal displacement $\mathrm{d} \boldsymbol{D}$ that is to be described in section 3.2, Equation (15) is rewritten as:

$$
\left(\frac{1}{\beta \Delta t^{2}} \boldsymbol{M}+\boldsymbol{K}^{(t+\Delta t)}-\left(\boldsymbol{J}^{(t+\Delta t)}\right)^{T} \boldsymbol{H}\right) \mathrm{d} \boldsymbol{D}=\boldsymbol{F}_{e r r}^{(t+\Delta t)}
$$


Solving this equation, we obtain $\mathrm{d} \boldsymbol{D}$ and accordingly $\mathrm{d} \ddot{\boldsymbol{D}}$ and $\mathrm{d} \boldsymbol{q}$. The values corresponding to the equation of motion at time $t+\Delta t$ are then corrected as:

$$
\begin{aligned}
& \boldsymbol{D}^{(t+\Delta t)} \leftarrow \boldsymbol{D}^{(t+\Delta t)}+\mathrm{d} \boldsymbol{D} \\
& \ddot{\boldsymbol{D}}^{(t+\Delta t)} \leftarrow \ddot{\boldsymbol{D}}^{(t+\Delta t)}+\mathrm{d} \ddot{\boldsymbol{D}} \\
& \boldsymbol{q}^{(t+\Delta t)} \leftarrow \boldsymbol{q}^{(t+\Delta t)}+\mathrm{d} \boldsymbol{q}
\end{aligned}
$$

On the basis of the updated displacement vector $\boldsymbol{D}^{(t+\Delta t)}$, the current system stiffness matrix $\boldsymbol{K}^{(t+\Delta t)}$ is updated. The force error vector is updated accordingly, and the convergence condition is evaluated by means of the following criterion:

$$
\frac{\mathrm{d} \boldsymbol{D}^{(\mu)} \cdot \boldsymbol{F}_{e r r}^{(t+\Delta t, \mu)}}{\mathrm{d} \boldsymbol{D}^{(1)} \cdot \boldsymbol{F}_{\text {err }}^{(t+\Delta t, 1)}}<\text { tolerance }
$$

where the 'tolerance' is a extremely small value that is set in advance by trial and error for different nonlinear dynamic problems. The iterative process between the time step $t$ and $t+\Delta t$ continues until the above condition is satisfied.

\subsection{Compatibility Condition of Truss Having SMA Wires}

The relationship between $\mathrm{d} \boldsymbol{q}$ and $\mathrm{d} \boldsymbol{D}$ is developed in this section. On the basis of the constitutive equation of SMA, the component of the $i$ th SMA member in vector $\mathrm{d} \boldsymbol{q}$ can be denoted as $\mathrm{d} q_{i}=E_{i} A_{i} \mathrm{~d} \epsilon_{i}^{t}$. As shown in Figure 2, the phase transformation strain of SMA is expressed as a piecewise linear function of total strain $\epsilon_{i}$ as:

$$
\epsilon_{i}^{t}=f\left(\epsilon_{i}\right)= \begin{cases}0 & \text { Part 1 } \\ \Lambda \frac{\epsilon_{i}-\epsilon_{m s}}{\epsilon_{m f}-\epsilon_{m s}} & \text { Part 2 } \\ \Lambda & \text { Part 3 } \\ \epsilon_{\max }^{t}-\epsilon_{\max }^{t} \frac{\epsilon_{C}-\epsilon_{i}}{\epsilon_{C}-\epsilon_{a s}} & \text { Part 4 } \\ \epsilon_{A}^{t} \text { or } \epsilon_{B}^{t} & \text { Part 5 }\end{cases}
$$

where parameters $\epsilon_{m s}$ and $\epsilon_{m f}$ are the martensite phase transformation start and finish strains, respectively; parameter $\epsilon_{\max }^{t}$ is the phase transformation strain at the beginning point of reverse phase transformation (point C); parameter $\epsilon_{C}$ is the total strain of point C; parameter $\epsilon_{a s}$ is the austenite phase transformation start strain; parameter $\epsilon_{A}^{t}$ and $\epsilon_{B}^{t}$ are the phase transformation strains of point $\mathrm{A}$ (the point at which the process of martensitic phase transformation stopped all of a sudden) and point B (the point at which the process of reversed phase transformation ceased suddenly), respectively. The incrementation of $\epsilon_{i}^{t}$ can be derived as:

$$
\mathrm{d} \epsilon_{i}^{t}=\frac{\partial f\left(\epsilon_{i}\right)}{\partial \epsilon_{i}} \mathrm{~d} \epsilon_{i}= \begin{cases}\frac{\Lambda}{\epsilon_{m f}-\epsilon_{m s}} \mathrm{~d} \epsilon_{i} & \text { Part } 2 \\ \frac{\epsilon_{\max }^{t}}{\epsilon_{C}-\epsilon_{a f}} \mathrm{~d} \epsilon_{i} & \text { Part } 4 \\ 0 & \text { Others }\end{cases}
$$

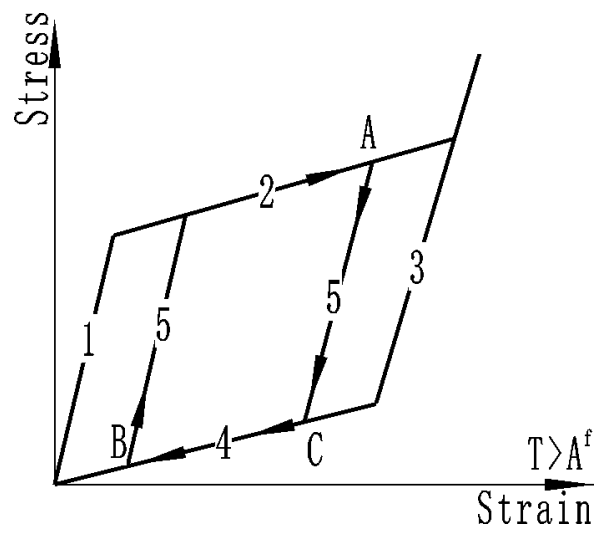

Figure 2. Piecewise analysis for phase transformation strain 
By denoting the deformation, the Jacobian matrix and the nodal displacement vector of the $i$ th SMA member as $r_{i}, \boldsymbol{J}^{e}$ and $\boldsymbol{D}^{e}$ respectively, the incrementation of the total strain of the $i$ th SMA member is attained as $\mathrm{d} \epsilon_{i}=\frac{\mathrm{d} r_{i}}{l_{i}}=\frac{\boldsymbol{J}^{e} \cdot \mathrm{d} \boldsymbol{D}^{e}}{l_{i}}$. By substituting $\mathrm{d} \epsilon_{i}$ into Equation (21), we can obtain the relationship between the incrementation of phase transformation strain and the incrementation of nodal displacement vector of the $i$ th SMA member:

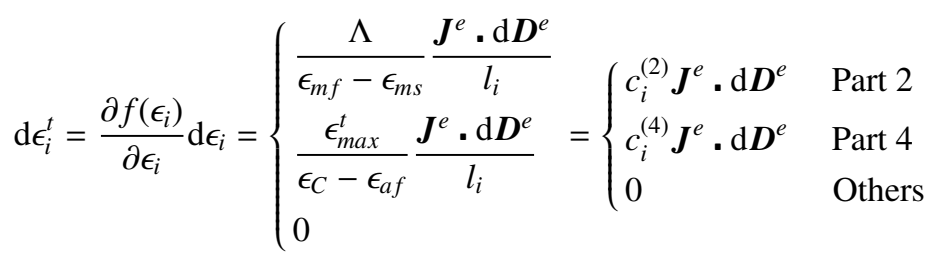

where coefficients $c_{i}^{(2)}$ and $c_{i}^{(4)}$ are denoted as one parameter $c_{i}$. On the above equations, we can express the $i$ th component in $\mathrm{d} q$ as:

$$
\mathrm{d} q_{i}=\left\{\begin{array}{lc}
E_{i} A_{i} \mathrm{~d} \epsilon_{i}^{t} & \text { SMA members } \\
0 & \text { Others }
\end{array}\right.
$$

On the basis of Equations (22) and (23), the following linear relation between $\mathrm{d} \boldsymbol{q}$ and $\mathrm{d} \boldsymbol{D}$ is achieved:

$$
\mathrm{d} \boldsymbol{q}=\boldsymbol{H} \mathrm{d} \boldsymbol{D}
$$

where $\boldsymbol{H}$ is the corresponding coefficient matrix obtained based on these equations.

\subsection{Numerical Integration Algorithm}

The numerical integration algorithm in order to deal with the dynamics of such kind of truss structure problem is carried out on the basis of the formulations in (Hanahara et al., 2016). This kind of dynamic problem is nonlinear due to the material nonlinearity of the SMA wires and geometric nonlinearity of the truss structure itself. This nonlinear iterative method is introduced in order to reduce the calculation time. It should be noted that, as the term $\left(\boldsymbol{J}^{(t+\Delta t)}\right)^{T} \mathrm{~d} \boldsymbol{q}$ is tremendously small, in the numerical calculation process, this term can be ignored, and there is little influence on the results.

\section{Simulation Examples}

Typical dynamic behaviors of hanging truss structural system with different configurations are discussed in order to demonstrate the effect of hanging configuration as well as the mechanical properties of SMA and ordinary wires.

\subsection{Assumed Truss Structural System and Condition of Environmental Vibration}

In Figure 3(a), the thick lines stand for rigid members, the broken lines stand for wire members. Numbers assigned to the broken lines shown in the figure serve as the identifiers of the wire members. In the simulation examples, the

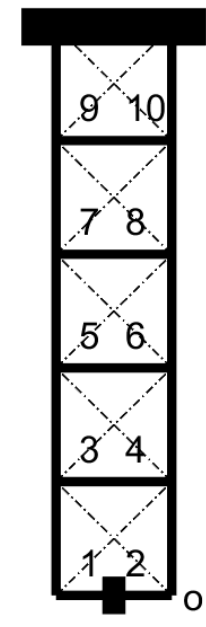

(a) Truss configuration

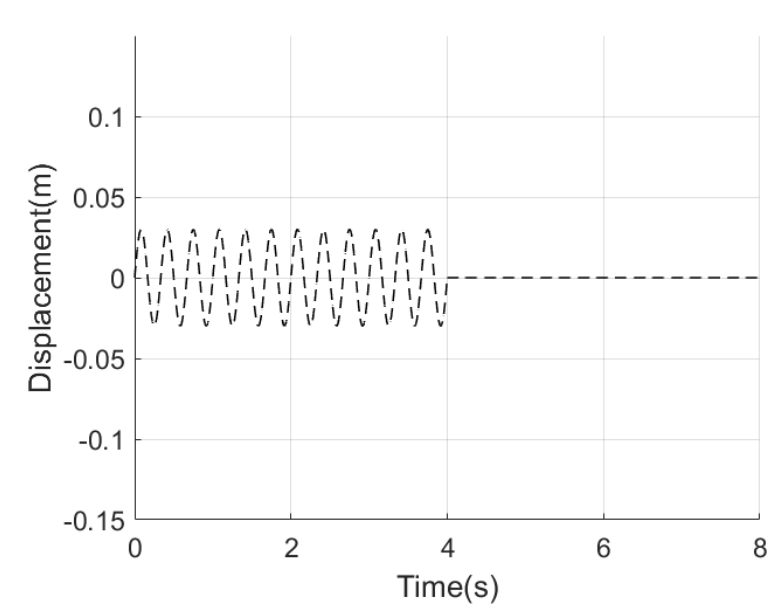

(b) Horizontal dynamic motion

Figure 3. Configuration of the truss and vibrational motion of support ceiling 


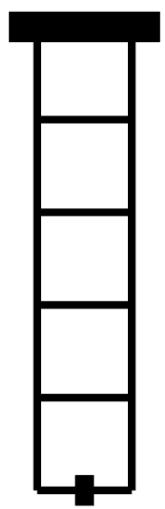

(a) No bracing wires

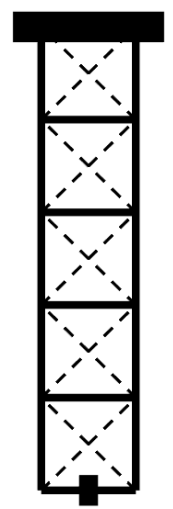

(b) 10 SMA wires

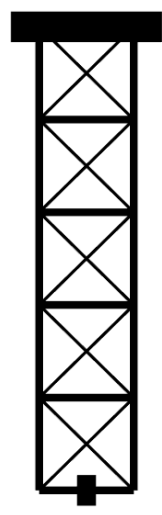

(c) 10 ordinary wires

Figure 4. Configurations of truss structures for the demonstration of influence of bracing member types

environmental motion is given in the form of vibrational motion of the support ceiling, whose corresponding displacement trajectory is sinusoidal shown as the waveform in Figure 3(b). The dynamic behavior in horizontal direction of the peripheral end node ' $\mathrm{o}^{\prime}$ shown in Figure 3(a) is to be examined. Corresponding parameters for the simulations and the characteristics of SMA wire are listed in Tables 1 and 2. In Table 1, the diameter of the ordinary wire is determined on the basis of the diameter of the SMA wire that is set in advance from the viewpoint of the same ultimate strength of those two kinds of wires. The vibration frequency of the support ceiling is $4 \mathrm{~Hz}$ and the amplitude is $0.03 \mathrm{~m}$. We assume an apparatus of $5 \mathrm{~kg}$ supported by the hanging truss. The total mass of the truss without the apparatus is $9.26 \mathrm{~kg}$; it should be noted that the mass is practically due to the mass of rigid members. The time step for the numerical integration is $50 \mu \mathrm{s}$. This tremendously small time step is for the purpose of dealing with the material nonlinearity and geometric nonlinearity of this kind of truss structural system.

\subsection{Demonstration of Vibration Isolation by Hanging Truss}

The following simulations are conducted to illustrate the vibration isolation capability of the hanging truss with the pendulum effect. In Figure 4(a), there are no bracing wires placed at the hanging truss. Therefore, this structure becomes a mechanism that is unstable if it is not in hanging configuration. However, under the influence of the gravitational force, stability of a structure of this type can be guaranteed in many situations.

Table 1. Simulation conditions

\begin{tabular}{llll}
\hline Content & Member & Parameter & Value \\
\hline Member diameter $(\mathrm{mm})$ & rigid & $a_{R}$ & $10 \mathrm{~mm}$ \\
& wire & $a_{w}$ & $1.6 \mathrm{~mm}$ \\
& SMA & $a_{\text {sma }}$ & $1 \mathrm{~mm}$ \\
Young's modulus $(\mathrm{GPa})$ & rigid & $E_{R}$ & $210 \mathrm{GPa}$ \\
& wire & $E_{w}$ & $210 \mathrm{GPa}$ \\
Density $\left(\mathrm{kg} / \mathrm{m}^{3}\right)$ & rigid & $\rho_{R}$ & $7860 \mathrm{~kg} / \mathrm{m}^{3}$ \\
& wire & $\rho_{w}$ & $7860 \mathrm{~kg} / \mathrm{m}^{3}$ \\
& SMA & $\rho_{\text {sma }}$ & $6500 \mathrm{~kg} / \mathrm{m}^{3}$ \\
\hline
\end{tabular}

Table 2. SMA characteristics

\begin{tabular}{lll}
\hline Name & Parameter & Value \\
\hline Maximum phase transformation strain & $\Lambda$ & 0.05 \\
Young's modulus of austenite phase & $E_{A}$ & $70 \mathrm{GPa}$ \\
Young's modulus of martensite phase & $E_{M}$ & $30 \mathrm{GPa}$ \\
\hline
\end{tabular}




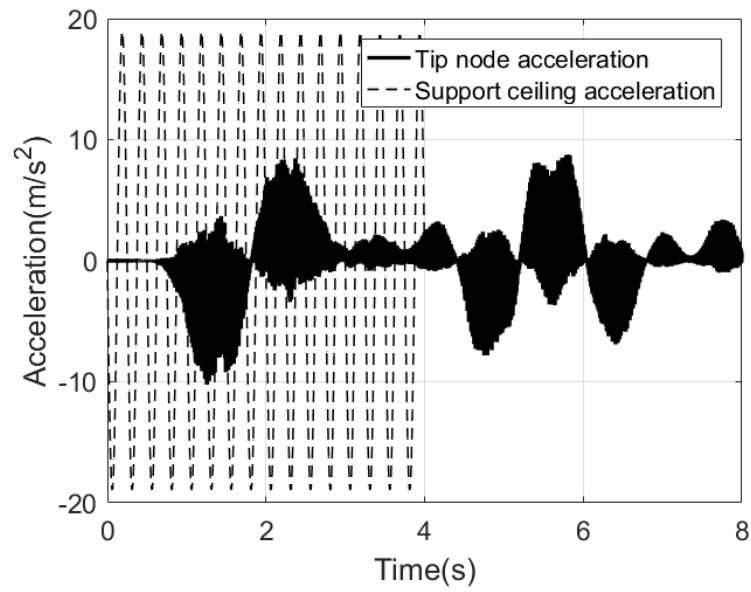

(a) $0 \mathrm{~kg}$

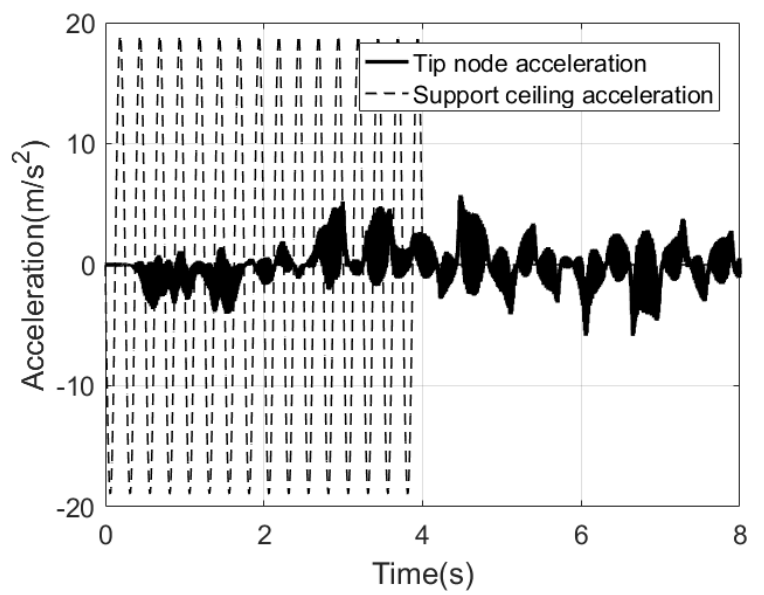

(c) $20 \mathrm{~kg}$

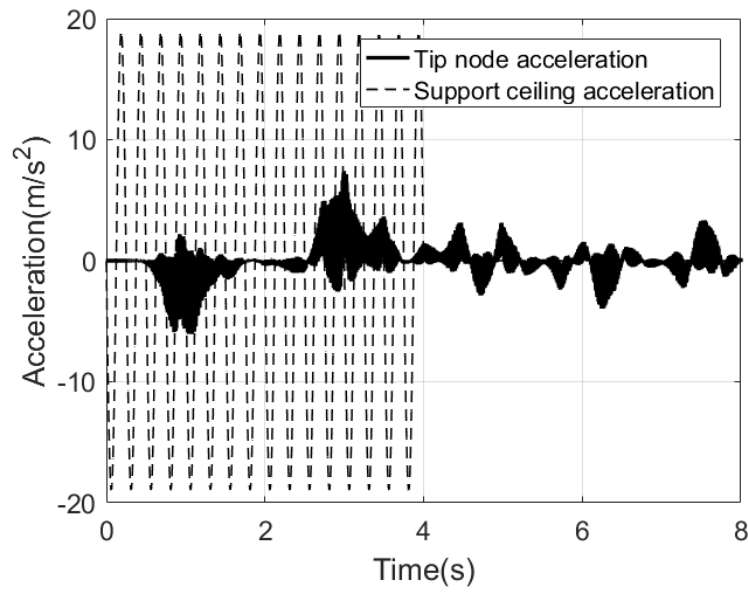

(b) $5 \mathrm{~kg}$

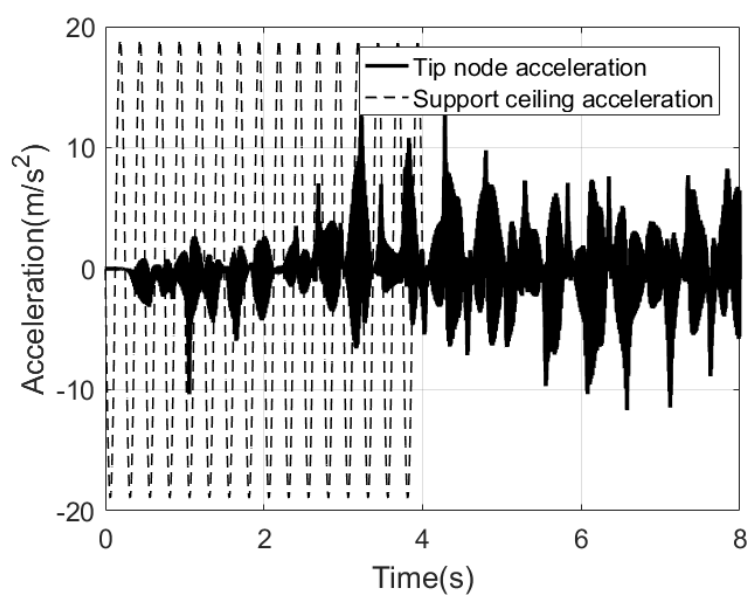

(d) $40 \mathrm{~kg}$

Figure 5. Dynamic behaviors of the truss in Figure 4(a) with different mass of the peripheral end apparatus

Figure 5 shows the corresponding dynamic behaviors of acceleration of the peripheral end node of the structure shown in Figure 4(a). In the four results of Figure 5, from the acceleration point of view, we confirmed the vibration isolation effect; the acceleration amplitude of the peripheral end is significantly smaller than the acceleration amplitude of the support ceiling. At the beginning of vibration, due to the influence of the inertia force, vibration isolation effect is obvious.

Figure 5 shows the influence on the dynamic behavior of the mass of the apparatus assumed at the peripheral end. By comparing the results in Figures 5(a)-5(c), we conclude the vibration isolation effect becomes obvious in accordance with the mass of the apparatus. The RMS value of the support ceiling vibrational motion is $9.47 \mathrm{~m} / \mathrm{s}^{2}$, and the RMS values of the results are listed in Table 3. The RMS values of acceleration of Figures 5(a), 5(b) and 5(c) are $2.35 \mathrm{~m} / \mathrm{s}^{2}, 1.29 \mathrm{~m} / \mathrm{s}^{2}$ and $1.53 \mathrm{~m} / \mathrm{s}^{2}$, respectively. These values illustrate the excellent vibration isolation capability of the truss structures evaluated in terms of acceleration when compared with the RMS value of the vibrational motion of the support ceiling. However, in the case of the mass of $40 \mathrm{~kg}$, the exerted vibration energy is significantly converted into the vibration of the peripheral end as shown in Figure 5(d). The RMS value of acceleration of Figure 5(d) is $2.77 \mathrm{~m} / \mathrm{s}^{2}$, and is obviously larger than the RMS values of Figures 5(a), 5(b) and 5(c), although the value is still smaller than that of the support ceiling. Therefore, the isolation effect can not be simply guaranteed with relatively large mass at the peripheral end of the hanging truss.

\subsection{Influence of Mechanical Properties of SMA and Ordinary Wire}

Figures 6 and 7 show the dynamic results that are adopted to demonstrate the influence of the mechanical properties of SMA and ordinary wires on the structural dynamic characteristics of the truss. Figures 6(a) and 6(b) are the displacement 


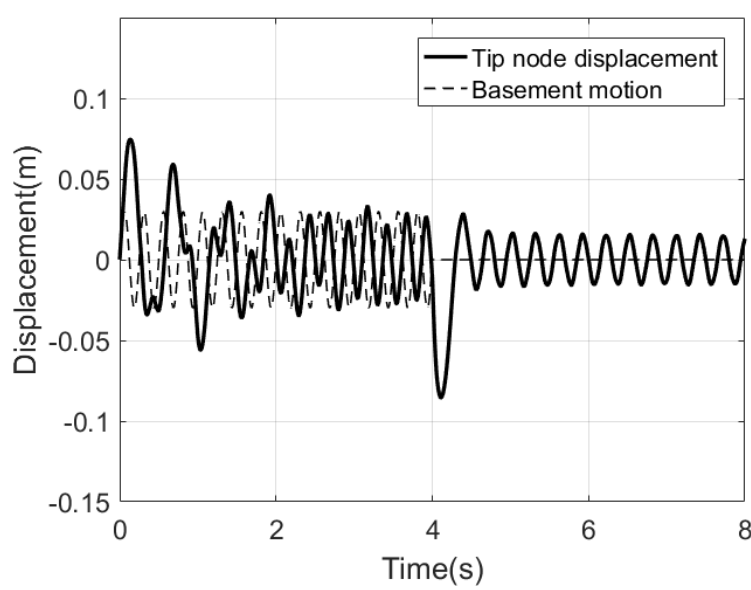

(a) Displacement behavior

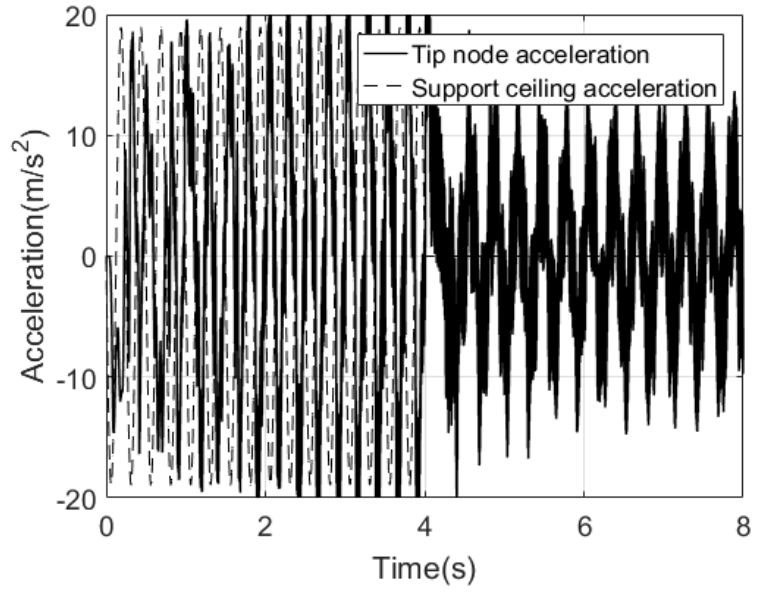

(b) Acceleration behavior

Figure 6. Dynamic behaviors of the truss in Figure 4(b)

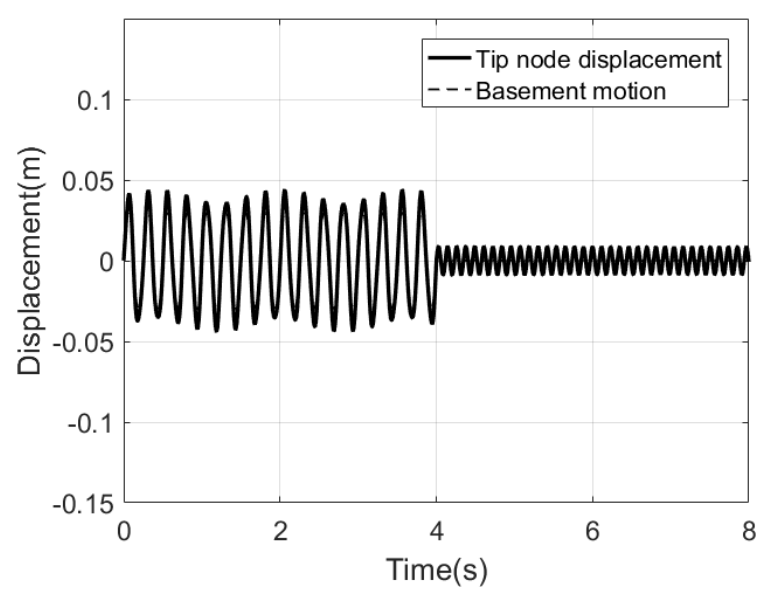

(a) Displacement behavior

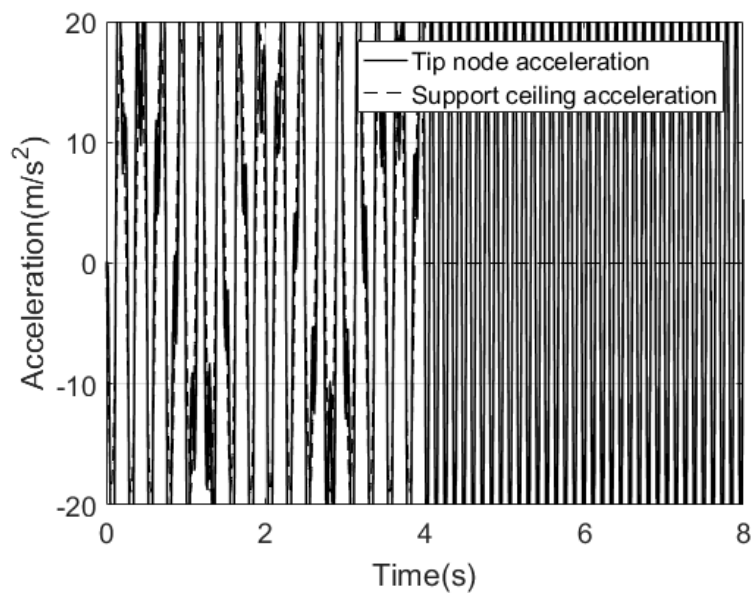

(b) Acceleration behavior

Figure 7. Dynamic behaviors of the truss in Figure 4(c)

and acceleration behaviors of the truss in Figure 4(b). In Figure 6(a), vibration isolation and attenuation effects due to SMA wires are demonstrated. When the support ceiling is vibrated, in the stage of transient response of 0 s-2s, the deformation of the truss is significant; in the stage of steady state of $2 \mathrm{~s}-4 \mathrm{~s}$, vibration isolation effect is demonstrated. At the time period of $4 \mathrm{~s}-8 \mathrm{~s}$, vibration attenuation effect due to the hysteretic loop of SMA wires can be seen. The RMS value of acceleration in Figure 6(b) is $9.31 \mathrm{~m} / \mathrm{s}^{2}$, which demonstrates that the vibration isolation of the truss with units having bracing of SMA wires is worse than the truss with units having no bracing wires.

Figures 7(a) and 7(b) are the displacement and acceleration behaviors of the truss in Figure 4(c). Since the ordinary wires have a higher stiffness than the SMA wires, the obtained behavior at time period of 0s-4s is also vibratory but relatively stable as shown in Figure 7(a), compared with the result shown in Figure 6(a). The RMS value of acceleration in Figure $7(\mathrm{~b})$ is $19.12 \mathrm{~m} / \mathrm{s}^{2}$, which is significantly larger than the case in Figure 6(b). However, the residual vibration amplitude in Figure 7(a) is smaller than the residual vibration amplitude in Figure 6(a). This is because the deformation of the truss structure having ordinary wires is smaller than the truss structure having SMA wires due to the relatively high stiffness of the ordinary wires.

Comparison between the two results in Figure 6(a) and 7(a), shows the obvious differences of the mechanical properties 
Table 3. RMS values of the acceleration results

\begin{tabular}{llll}
\hline Type & Mass $(\mathrm{kg})$ & Result & RMS $\left(\mathrm{m} / \mathrm{s}^{2}\right)$ \\
\hline No bracing wires & $0 \mathrm{~kg}$ & $5(\mathrm{a})$ & 2.35 \\
No bracing wires & $5 \mathrm{~kg}$ & $5(\mathrm{~b})$ & 1.29 \\
No bracing wires & $20 \mathrm{~kg}$ & $5(\mathrm{c})$ & 1.53 \\
No bracing wires & $40 \mathrm{~kg}$ & $5(\mathrm{~d})$ & 2.77 \\
10 SMA wires & $5 \mathrm{~kg}$ & $6(\mathrm{~b})$ & 9.31 \\
10 ordinary wires & $5 \mathrm{~kg}$ & $7(\mathrm{~b})$ & 19.12 \\
4 SMA wires & $5 \mathrm{~kg}$ & $9(\mathrm{~b})$ & 1.57 \\
\hline
\end{tabular}

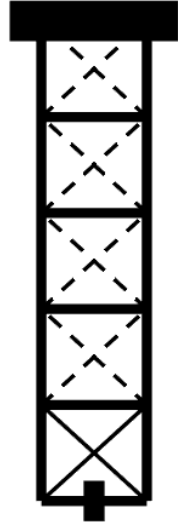

(a) Truss

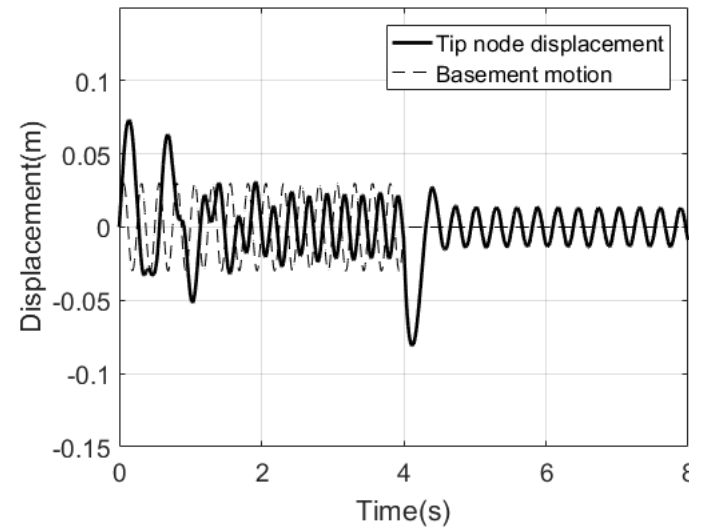

(b) Displacement behavior

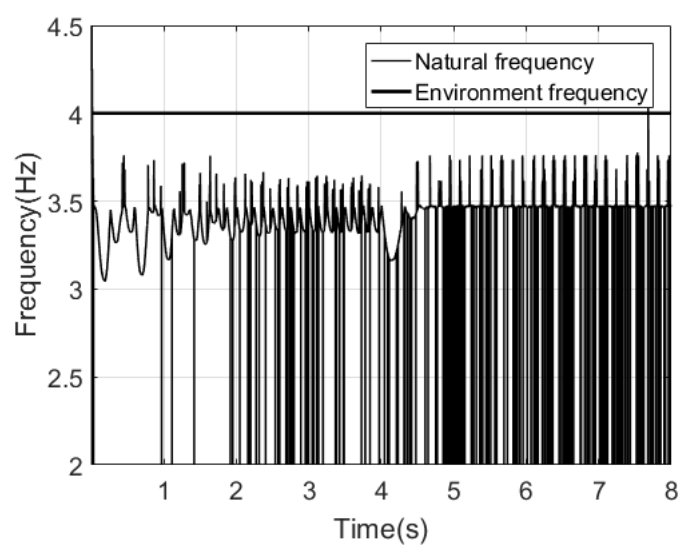

(c) Time history of natural frequency

Figure 8. Dynamic behaviors of the truss having 8 SMA wires

between the two kinds of wires. Combination of these two types of wires with different mechanical properties is expected to enable the structure to improve vibration attenuation as well as vibration isolation capabilities simultaneously.

\subsection{Combination of SMA and Ordinary Wires}

Hysteretic loop and variable stiffness characteristics of SMA wires enable the truss structure to have the capabilities of vibration attenuation and isolation. In the case of low strain range (without phase transformation) and slack state, such kind of SMA wires can be replaced by ordinary wires; simultaneously, owing to the relatively high mechanical stiffness of the ordinary wires, stability of the peripheral end can be maintained to some extent from the deformation point of view.

For instance, Figure 8(a) is a truss structure having 8 SMA wires and 2 ordinary wires. Figure $8(\mathrm{~b})$ is the corresponding displacement behavior of the peripheral end node in horizontal direction. This result demonstrates vibration isolation effect in case that support ceiling vibrated, as well as vibration attenuation effect in case that support ceiling vibration ceased.

Figure 8(c) is the corresponding time history of the natural frequency of the truss structure in Figure 8(a). The thin line represents the time history of the natural frequency, and the thick line indicates the environmental vibration frequency of the support ceiling. The initial natural frequency is $3.47 \mathrm{~Hz}$. In the transitional period of $0 \mathrm{~s}-2 \mathrm{~s}$, the natural frequency changes drastically as the mechanical properties of the SMA wires; in the stage of steady state of $2 \mathrm{~s}-4 \mathrm{~s}$, natural frequency changes periodically, which demonstrates the variable stiffness of truss structural system as a whole. It should be noted that in the calculation process, in the case that the two wires at the same truss unit are in slack state or in taut state simultaneously, the natural frequency of the truss becomes extremely small or big suddenly. The slack possibility of wire, the variable stiffness of SMA and relatively small stiffness property of wire members contribute to the vibration isolation effect of the truss structure. The RMS value of displacement in Figure $8(\mathrm{~b})$ is $0.022 \mathrm{~m}$, which is smaller than the RMS value of displacement in Figure 6(a), which is $0.026 \mathrm{~m}$. These RMS values corresponding to the truss structures in Figure 4(b) and 8(a) indicate that an appropriate combination of SMA wires and ordinary wires plays an important role in the 
ability of truss structural system against environmental vibration from the vibration isolation point of view in the case of being evaluated in terms of displacement criterion.

\subsection{Combination of Units Having No Bracing Wires and Units Having SMA wires}

Hanging configuration of unstable mechanism have the effect that it can isolate its peripheral end from the support ceiling vibration under the vibration condition of small amplitude and relatively high frequency. Combination of unstable mechanism in hanging configuration and the mechanical characteristics of SMA wire is expected to improve this vibration isolation capability. The following example is adopted to demonstrate this phenomenon.

The result in Figure 9(b) is the acceleration behavior of the truss in Figure 9(a). In order to make a comparison in the time period of $0 \mathrm{~s}-4 \mathrm{~s}$ between the truss in Figure 4(a) and the truss in Figure 9(a), the same vibration condition is adopted. In addition, the configuration is the same as well except for the 4 SMA wires in Figure 9(a). We discuss the vibration isolation effect of $0 \mathrm{~s}-4 \mathrm{~s}$ in the results shown in Figures 5(b) and 9(b). The RMS values of the time period of 0s-4s in Figure 5 (b) and 9 (b) are $1.57 \mathrm{~m} / \mathrm{s}^{2}$ and $1.45 \mathrm{~m} / \mathrm{s}^{2}$, respectively. These two values illustrate that with appropriate arrangement of SMA wires, the vibration isolation effect can be improved.

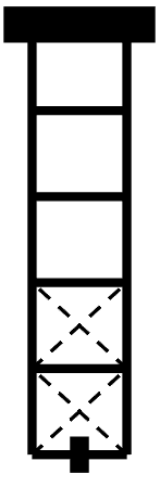

(a) Configuration

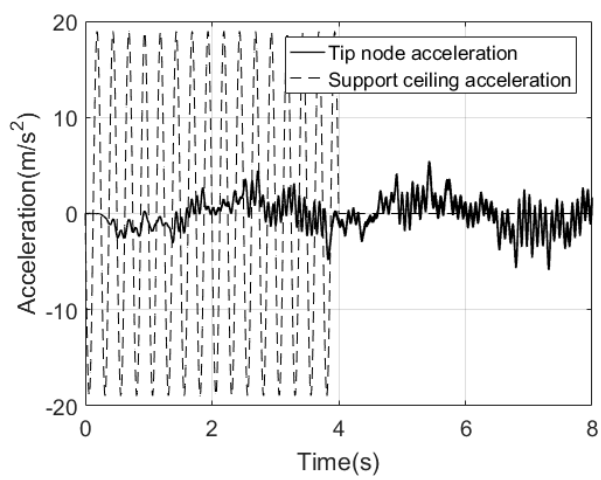

(b) Dynamic behavior of the truss

Figure 9. Dynamic behaviors of the truss having 4 SMA wires

\section{Conclusion}

Truss structures having SMA wires in hanging configuration possess the capabilities of vibration attenuation and vibration isolation based on the outstanding characteristics of the pseudo-elasticity of the SMA wires and the hanging configuration itself. Relatively high stiffness of the ordinary elastic wire contributes to the relative stability of the dynamic behaviors of the truss structure. Mathematical model of this kind of problem is proposed, and the corresponding numerical integration method is introduced. We have demonstrated these capabilities of vibration isolation and attenuation of the truss structure by simulation calculations with various kinds of combinations of units having bracing of SMA wires, ordinary wires and units having no bracing wires.

On the basis of simulation results, we have shown that, with appropriate combinations of units having bracing of SMA wires, ordinary wires, and units having no bracing wires, the vibration isolation capability of the truss structure can be improved due to the hanging configuration effect of the truss as well as the mechanical characteristics of SMA wire and ordinary wire. The time history of the natural frequency of the truss structure having SMA wire and ordinary wire demonstrates the variable stiffness of the truss structural system, which shows that variable stiffness of wire members contribute to the vibration isolation effect of the dynamic behaviors.

\section{References}

Akhverdiev, K. S., \& Mukutadze, A. M. (2016). Damper with Porous Anisotropic Ring. Mechanical Engineering Research, 6(2), 1-10. https://doi.org/10.5539/mer.v6n2p1

Ali, N. B. H., \& Smith, I. F. C. (2010). Dynamic Behavior and Vibration Control of a Tensegrity Structure. International Journal of Solids and Structures, 47(9), 1285-1296. http://doi.org/10.1016/j.ijsolstr.2010.01.012

Auricchio, F., \& Sacco, E. (1997). A One-dimensional Model for Superelastic Shape-memory Alloys with Different Elastic Properties between Austenite and Martensite. International Journal of Non-linear Mechanics, 32(6), 11011114. https://doi.org/10.1016/S0020-7462(96)00130-8 
De Lima, A. M. G., Guaraldo-Neto, B., Sales, T. P., \& Rade, D. A. (2014). A Time-domain Modeling of Systems Containing Viscoelastic Materials and Shape Memory Alloys as Applied to the Problem of Vibration. Engineering Structures, 68, 85-95. https://doi.org/10.1016/j.engstruct.2014.02.035

dos Santos, F. A., Rodrigues, A., \& Micheletti, A. (2015). Design and Experimental Testing of an Adaptive ShapeMorphing Tensegrity Structure, with Frequency Self-Tuning Capabilities, Using Shape-Memory Alloys. Smart Materials and Structures, 24(10), 105008. http://iopscience.iop.org/0964-1726/24/10/105008

Hanahara, K., Zhang, X., \& Tada, Y. (2016). Dynamic Simulation of Adaptive Truss Consisting of Various Types of Truss Members. Mechanical Engineering Research, 6(1), 75-87. http://dx.doi.org/10.5539/mer.v6n1p75

Hu, J. W. (2013). Numerical Simulation for the Behavior of Superelastic Shape Memory Alloys. Journal of Mechanics Science and Technology, 27(2), 381-386. https://doi.org/10.1007/s12206-012-1268-8

Lagoudas, D. C., Mayes, J. J., \& Khan, M. M. (2001). Simplified Shape Memory Alloy (SMA) Material Model for Vibration Isolation. Smart Structures and Materials 2001: Modeling, Signal Processing, and Control in Smart Structures. http://dx.doi.org/10.1117/12.436514

McCormick, J., DesRoches, R., Fugazza, D., \& Auricchio, F. (2010). Seismic Vibration Control Using Superelastic Shape Memory Alloys. Journal of Engineering Materials and Technology, 128(3), 294-301. https://doi.org/10.1115/ 1.2203109

Mortazavi, S. M. R., Ghassemieh, M., \& Motahari, S. A. (2013). Seismic Control of Steel Structures with Shape Memory Alloys. International Journal of Automation and Control Engineering, 2(1), 28-34.

Narita, R., Kojima, T., Kuramochi, H., Toriya, T., Kitayama, N., Kuramochi, M., \& Ohtsuka, T. (2000). Evaluation of Performance of Multi-Suspended Pendulum Isolation (MPI) Systme with Spatial Truss Structure. 12th World Conference on Earthquake Engineering.

Omar, M. (2014). Seismic Response of Braced Steel Frames with Shape Memory Alloy and Mega Bracing Systems. International Journal of Civil, Architectural Science and Engineering, 8(2), 131-138.

Patil, S. S., \& Awasare, P. J. (2016). Vibration Isolation of Lumped Masses Supported on Beam by Imposing Nodes Using Multiple Vibration Absorbers. Mechanical Engineering Research, 6(1), 88-95. http://dx.doi.org/10.5539/mer. v6n 1 p88

Sanap, S., Jadhal, P. D., \& Dumne, S. M. (2014). Earthquake Response Mitigation of RC Building Using Friction Pendulum System. American Journal of Engineering Research, 3(11), 30-37. http://ajer.org/papers/v3(11)/D0311030037

Senba, A., Oka, K., Takahama, M., \& Furuya, H. (2009). Vibration Reduction by Natural Frequency Optimization for Manipulation of a Variable Geometry Truss. 8th World Congress on Structural and Multidisciplinary Optimization.

Song, G., Ma, N., \& Li, H. N. (2006). Applications of Shape Memory Alloys in Civil Structures. Engineering Structures, 28(9), 1266-1274. https://doi.org/10.1016/j.engstruct.2005.12.010

Tamai, H., \& Kitagawa, Y. (2002). Pseudoelastic Behavior of Shape Memory Alloy Wire and Its Application to Seismic Resistance Member for Building. Computational Materials Science, 25(1), 218-227. https://doi.org/10.1016/ S0927-0256(02)00266-5

Tatemichi, I., Kawaguchi, M., \& Abe, M. (2004). A Study on Pendulum Seismic Isolators for High-Rise Buildings. The 2004 Council on Tall Buildings and Urban Habitat (CTBUH) Conference.

Thai, H. T., \& Kim, S. E. (2011). Nonlinear Inelastic Time-History Analysis of Truss Structures. Journal of Constructional Steel Research, 67(12), 1966-1972. https://doi.org/10/1016/j.jcsr/2011.06.015

Yang, C. S. W., DesRoches, R., \& Leon, R. T. (2010). Design and Analysis of Braced Frames with Shape Memory Alloy and Energy-absorbing Hybrid Devices. Engineering Structures, 32(2), 498-507. https://doi.org/10.1016/j.engstruct. 2009.10.011

\section{Copyrights}

Copyright for this article is retained by the author(s), with first publication rights granted to the journal.

This is an open-access article distributed under the terms and conditions of the Creative Commons Attribution license (http://creativecommons.org/licenses/by/4.0/). 